\title{
The magnetic field in luminous star-forming galaxies
}

\author{
Timothy Robishaw ${ }^{1}$ and Carl Heiles ${ }^{2}$ \\ ${ }^{1}$ Sydney Institute for Astronomy, School of Physics, The University of Sydney \\ email: robishaw@physics.usyd.edu.au \\ ${ }^{2}$ Astronomy Department, University of California, Berkeley \\ email: heiles@astro.berkeley.edu
}

\begin{abstract}
An ongoing search for Zeeman splitting in the $1667 \mathrm{MHz} \mathrm{OH}$ megamaser emission from luminous star-forming galaxies has yielded numerous detections. These results, in addition to being the first extragalactic measurement of the Zeeman effect in an emission line, suggest that $\mathrm{OH}$ megamasers are excellent extragalactic magnetometers. We review the progress of our survey and discuss future observations.
\end{abstract}

Keywords. Masers - galaxies: magnetic fields - galaxies: starburst - ISM: molecules

\section{What we knew then}

The study of magnetic fields in luminous star-forming galaxies has been limited to optical, infrared, and submillimeter polarimetric imaging of the nearby starburst galaxy M82. This process is sensitive to the plane-of-sky component of the magnetic field and does not permit the estimation of field strengths. Using this method, Jones (2000) was able to infer that the field in the nucleus of M82 is configured in a polar geometry aligned with the bipolar superwind seen to be emanating from the nuclear region, while the field in the disk of the galaxy is arranged in a toroidal configuration.

Wielebinski (2006) used the $100 \mathrm{~m}$ Effelsberg radio telescope to map the plane-of-sky magnetic field in M82 using polarized $32 \mathrm{GHz}$ emission. These observations confirmed the azimuthal field structure in the disk and vertical field structure along the poles but provide no estimate of the magnetic field strength in M82.

To the knowledge of the authors, no direct field strength measurements had been made towards nearby luminous star-forming galaxies until the results of Robishaw, Quataert, \& Heiles (2008), who needed a new tool to help them.

\section{ULIRGs and $\mathrm{OH}$ Megamasers}

Ultraluminous infrared galaxies (ULIRGs) are a population of galaxies that emit farinfrared (FIR) radiation with energies comparable to those of the most luminous quasars $\left(\log \left(L_{\mathrm{FIR}} / L_{\odot}\right)>12\right)$. Nearly every ULIRG appears to have undergone a merger or interaction and contains massive star formation and/or an active galactic nucleus (AGN) induced by gravitational interactions. Diamond et al. (1999) conducted very long baseline interferometry (VLBI) observations of the $1667 \mathrm{MHz}$ hydroxyl (OH) transition in the nuclear regions in ULIRGs and found multiple masing regions with $1<\log \left(L_{\mathrm{OH}} / L_{\odot}\right)<4$; these regions are known as $\mathrm{OH}$ megamasers (OHMs). Each OHM has a spectral linewidth of between 50 and $150 \mathrm{~km} \mathrm{~s}^{-1}$; when viewed by a single dish, these spectral components are superimposed. The $1667 \mathrm{MHz}$ OHM flux is always a few to many times the flux of the $1665 \mathrm{MHz}$ transition and in many cases the $1665 \mathrm{MHz}$ line is absent (Darling \& Giovanelli 
2002); this is an interesting contrast to the case of OH masers in the Galaxy in which the $1665 \mathrm{MHz}$ is usually the dominant transition (Reid \& Moran 1988). The starbursts and AGNs in ULIRGs create strong FIR dust emission as well as a strong radio continuum; the OHMs are generally believed to be pumped by the FIR radiation field (e.g., Randell et al. 1995) although collisional excitation may be important as well (e.g., Lonsdale et al. 1998). Given the conditions that exist in ULIRGs and considering that every OH maser in our Galaxy is associated with massive star-forming regions, it is not surprising that the entire OHM sample finds homes in LIRGs, strongly favoring the most FIR-luminous, the ULIRGs (Darling \& Giovanelli 2002).

ULIRGs are natural locations to expect very strong magnetic fields given their high gas and energy densities. Much of the radio emission in ULIRGs is resolved on scales $\sim 100$ pc with VLA observations (Condon et al. 1991). In Arp 220, high-resolution observations (Rovilos et al. 2003) show that the OHMs arise in this region as well. With this size scale and the observed radio fluxes, minimum energy arguments suggest volume averaged field strengths of $\approx 1 \mathrm{mG}$ (e.g., Condon et al. 1991, Thompson et al. 2006), significantly larger than the $\sim 10 \mu \mathrm{G}$ fields measured in normal spirals. The field strengths in ULIRGs cannot be much below a $\mathrm{mG}$ or else inverse Compton cooling would dominate over synchrotron cooling, making it energetically difficult to explain the radio fluxes from ULIRGs and the fact that ULIRGs lie on the FIR-radio correlation. The field strengths could, however, in principle be larger than the minimimum energy estimate if, as in our Galaxy, the magnetic energy density is in approximate equipartition with the total pressure (Thompson et al. 2006). This can be estimated from the observed surface density; CO observations of Arp 220 and several other systems reveal $\sim 10^{9} M_{\odot}$ of molecular gas in the central $\sim 100 \mathrm{pc}$ (e.g., Downes \& Solomon 1998) implying gas surface densities $\Sigma \sim 1-10 \mathrm{~g} \mathrm{~cm}^{-2}, 10^{3}-10^{4}$ times larger than in the Milky Way. The equipartition field scales as $B \propto \Sigma$ implying that the mean field in ULIRGs could approach $\sim 10 \mathrm{mG}$.

\section{Observations}

Motivated by the above considerations, Robishaw, Quataert, \& Heiles (2008) used both the $300 \mathrm{~m}$ Arecibot telescope and the $100 \mathrm{~m}$ Robert C. Byrd Green Bank Telescope $\ddagger$ in full-Stokes mode in an attempt to detect Zeeman splitting in megamaser emission of the $1667 \mathrm{MHz} \mathrm{OH}$ transition. Eight of the brightest OHMs were observed by spending equal time at on-source and off-source positions. Every VLBA observation of OHM emission in ULIRGs has shown that the spatial extent of the masing spots is of order at most a few arc seconds, much smaller than the beam of either Arecibo or the GBT; there are no instrumental polarization effects due to beam structure or sidelobes. The observations were made in full-Stokes mode and the Mueller matrix was calibrated using the standard technique of observing the linearly-polarized continuum sources 3C 138 and 3C 286 (Heiles et al. 2001, Heiles \& Troland 2004).

\section{What we know now}

Five of the eight sources that we observed exhibit significant Stokes $V$ emission that can be interpreted as Zeeman splitting of the $1667 \mathrm{MHz}$ line. We present the results for the

$\dagger$ The Arecibo Observatory is part of the National Astronomy and Ionosphere Center, which is operated by Cornell University under a cooperative agreement with the National Science Foundation.

$\ddagger$ The National Radio Astronomy Observatory is a facility of the National Science Foundation operated under cooperative agreement by Associated Universities, Inc. 
canonical ULIRG Arp 220 in the left panels of figure 1; the right panels of figure 1 show the results for III Zw 35. The top panel for each source shows the Stokes $I$ spectrum 9 as a function of frequency and optical heliocentric velocity. It is clear that the OHM emission profile for each source is a composite of many narrower components.
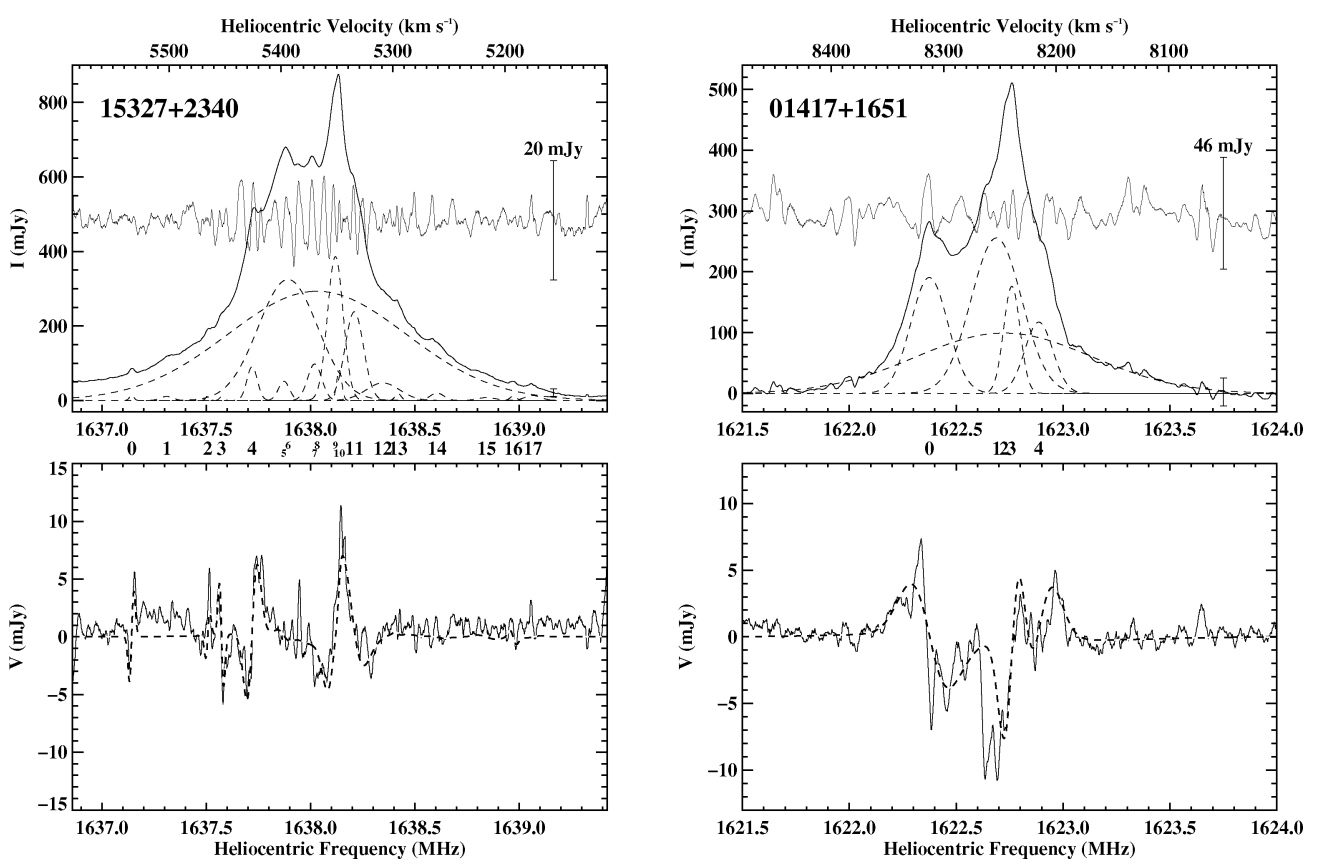

Figure 1. Stokes I spectrum (top left) for Arp 220. The profile of each Gaussian component is plotted as a dashed line. The residuals of the composite Gaussian profile from the data are plotted on an enhanced scale through the center of the Stokes I plot. The Stokes $V$ (bottom left) spectrum of Arp 220; the composite fit for Zeeman splitting of each corresponding Gaussian component is plotted as a dashed line. (Top right) Stokes I profile for III $\mathrm{Zw}$ 35. (Bottom right) Stokes $V$ spectrum for III $\mathrm{Zw} 35$.

\subsection{Arp 220}

The Stokes $I$ profile was decomposed into 18 Gaussian components; we attempted to use the fewest number of components to obtain reasonable residuals (which are plotted with an enhanced scale through the middle height of the Stokes $I$ profile) while allowing enough components to reproduce multiple splittings in the Stokes $V$ spectrum. The complexity of the VLBA spectra of Rovilos et al. (2003) prevents a clear association of single-dish components with the individual maser spots. The bottom panel shows the Stokes $V$ spectrum: it is clear that the detectable circular polarization corresponds well with many of the individual Stokes $I$ peaks. Six components show Zeeman signatures in the Stokes $V$ spectrum and the absolute values of the fitted magnetic field strengths range from 0.7 to $4.7 \mathrm{mG}$. The magnetic field reverses within Arp 220 since four fields point towards us and two away.

I We use the classical definition of Stokes $I$, which is the sum (not the average) of two orthogonal polarizations. Thus, fluxes in Stokes $I$ are twice the usual flux density given in catalogs. 


\subsection{III $Z w 35$}

The Stokes $I$ profile for III Zw 35 required only 5 Gaussian components to obtain reasonable residuals. Pihlström et al. (2001) and Parra et al. (2005) model high-resolution observations of the $\mathrm{OHM}$ emission as a torus of multiple maser clouds inclined at an angle of $60^{\circ}$ and rotating at $57 \mathrm{~km} \mathrm{~s}^{-1}$ away from us at the north and towards us at the south.

From the circular polarization spectrum, components 0,3 , and 4 display fields of +2.94 , -2.73 , and $-3.59 \mathrm{mG}$, respectively. From the high-resolution mapping of Pihlström et al. (2001), component 0 appears to be associated with the northern maser spots, while components 3 and 4 seem to originate in the south. In terms of the torus model, these results suggest that the magnetic field might be constrained to the OHM torus with the magnetic field following the velocity field, pointing away from us in the north and towards us in the south.

\subsection{Summary of results}

Robishaw, Quataert, \& Heiles (2008) report a total of 14 independent field detections in multiple components within five extragalactic sources including Arp 220 and III Zw 35. The median absolute field strength is $\sim 3 \mathrm{mG}$ and the direction of the field reverses within 3 of the galaxies. The distribution is strikingly similar to that in the $\mathrm{OH}$ masers that probe the star-forming regions in the Milky Way (Fish et al. 2003), suggesting that the conditions in regions of massive star formation in ULIRGs are similar to the conditions in our own Galaxy's star-forming regions. The measured magnetic field strengths are also consistent with those inferred from sychrotron emission in ULIRGs by Thompson et al. (2006).

\section{OH Megamasers: the new extragalactic magnetometers}

The Zeeman detection rate for our sample of ULIRGs suggests that OHMs are excellent extragalactic magnetometers. Since it is not possible to unambiguously associate VLBI spectra of the OHM spots within any ULIRG with the Gaussian components in our single-dish spectra, it will be necessary to follow up all detections with full-Stokes VLBI observations in order to detect Zeeman splitting directly in the OHM spots. This would allow us to make high-resolution maps of the magnetic field strength and line-of-sight field direction in star-forming galaxies. Such high-resolution field maps would allow us to infer whether the field structure in the nuclei of these starburt galaxies is similar to that of M82.

A full-Stokes, flux-limited Arecibo survey of OHMs (including the $\mathrm{OH}$ satellite lines) is currently underway at Arecibo. Additional Zeeman detections have been revealed in the preliminary results. Finally, we have proposed to carry our hunt for Zeeman splitting to the southern OHM population by incorporating the $64 \mathrm{~m}$ Parkes telescope.

\section{Acknowledgements}

This research was supported in part by NSF grant AST-0406987. Support for this work was also provided to TR by the NSF through award GSSP 06-0003 from the NRAO. TR would like to thank: the SOC for an invitation to present these results, the IAU for providing financial support to attend this conference, and Guayota for not becoming angry. 


\section{References}

Condon, J. J., Huang, Z.-P., Yin, Q. F., \& Thuan, T. X. 1991, ApJ 378, 65

Darling, J. \& Giovanelli, R. 2002, AJ 124, 100

Diamond, P. J., Lonsdale, C. J., Lonsdale, C. J., \& Smith, H. E. 1999, ApJ 511, 178

Downes, D. \& Solomon, P. M. 1998, ApJ 507, 615

Fish, V. L., Reid, M. J., Argon, A. L., \& Menten, K. M. 2003, ApJ 596, 328

Heiles, C., et al. 2001, PASP 113, 1274

Heiles, C. \& Troland, T. H. 2004, ApJS 151, 271

Jones, T. J. 2000, $A J$ 120, 2920

Lonsdale, C. J., Lonsdale, C. J., Diamond, P. J., \& Smith, H. E. 1998, ApJL 493, L13

Parra, R., Conway, J. E., Elitzur, M., \& Pihlström, Y. M. 2005, A\& $A$ 443, 383

Pihlström, Y. M., Conway, J. E., Booth, R. S., Diamond, P. J., \& Polatidis, A. G. 2001, A\&A 377,413

Randell, J., Field, D., Jones, K. N., Yates, J. A., \& Gray, M. D. 1995, A\&A 300, 659

Reid, M. J. \& Moran, J. M. 1988, in Galactic and Extragalactic Radio Astronomy, ed. G. .L. Verschuur \& K. I. Kellermann (2nd ed.; Berlin: Springer), 255

Robishaw, T., Quataert, E., \& Heiles, C. 2008, ApJ 680, 981

Rovilos, E., Diamond, P. J., Lonsdale, C. J., Lonsdale, C. J., \& Smith, H. E. 2003, MNRAS 342,373

Thompson, T. A., Quataert, E., Waxman, E., Murray, N., \& Martin, C. L. 2006, ApJ 645, 186

Wielebinski, R. 2006, $A N$ 327, 510

\section{Discussion}

SARMA: From the I profiles, one can see that several masers are present in the Arecibo beam, and unresolved. Is it the expectation that VLBA observations will spatially resolve these into single maser "spots"?

RoBISHAw: Yes! Only a handful of the brightest OHMs have been imaged with VLBI. In most cases, the VLB maps recover all of the single-dish flux. For the measured milligauss field strengths in these OHMs, we should be able to directly map the field in these ULIRGs in a reasonable integration time by directly detecting the Zeeman splitting of the megamaser spots using the high-sensitivity VLBA.

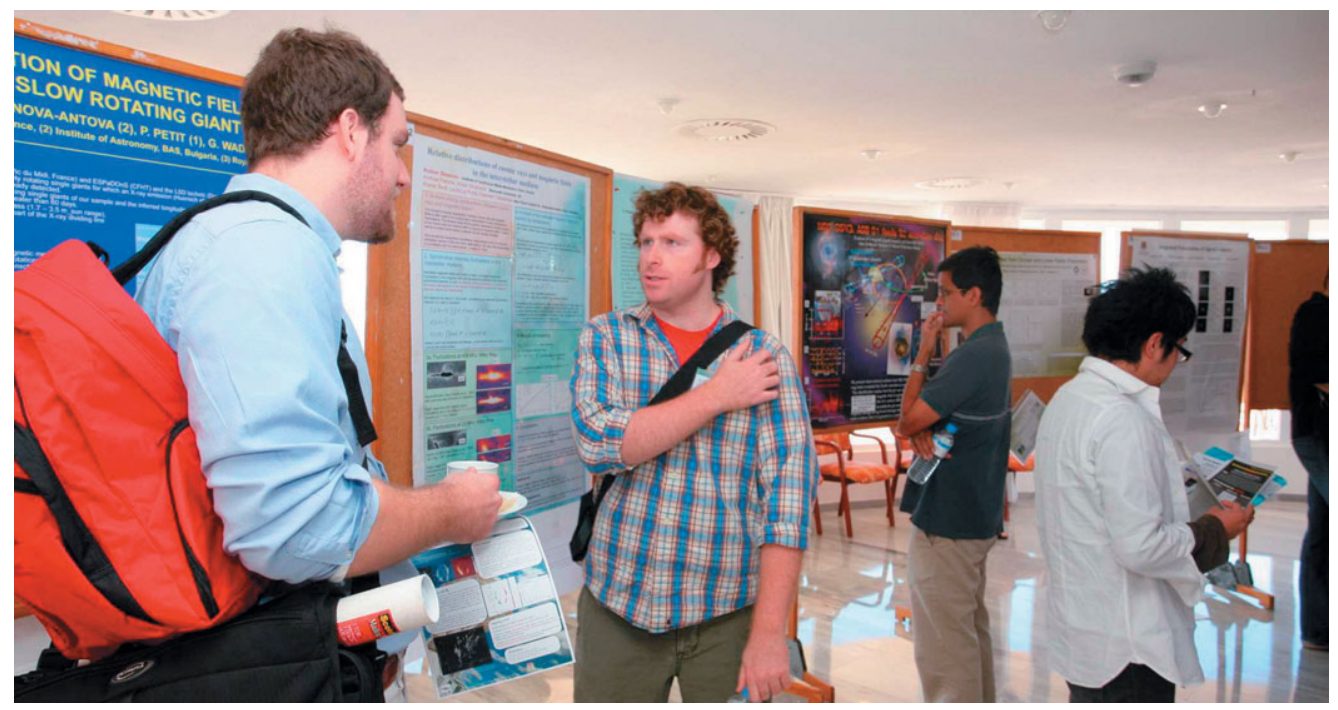

Shea Brown (left), and Tim Robishaw (middle) 


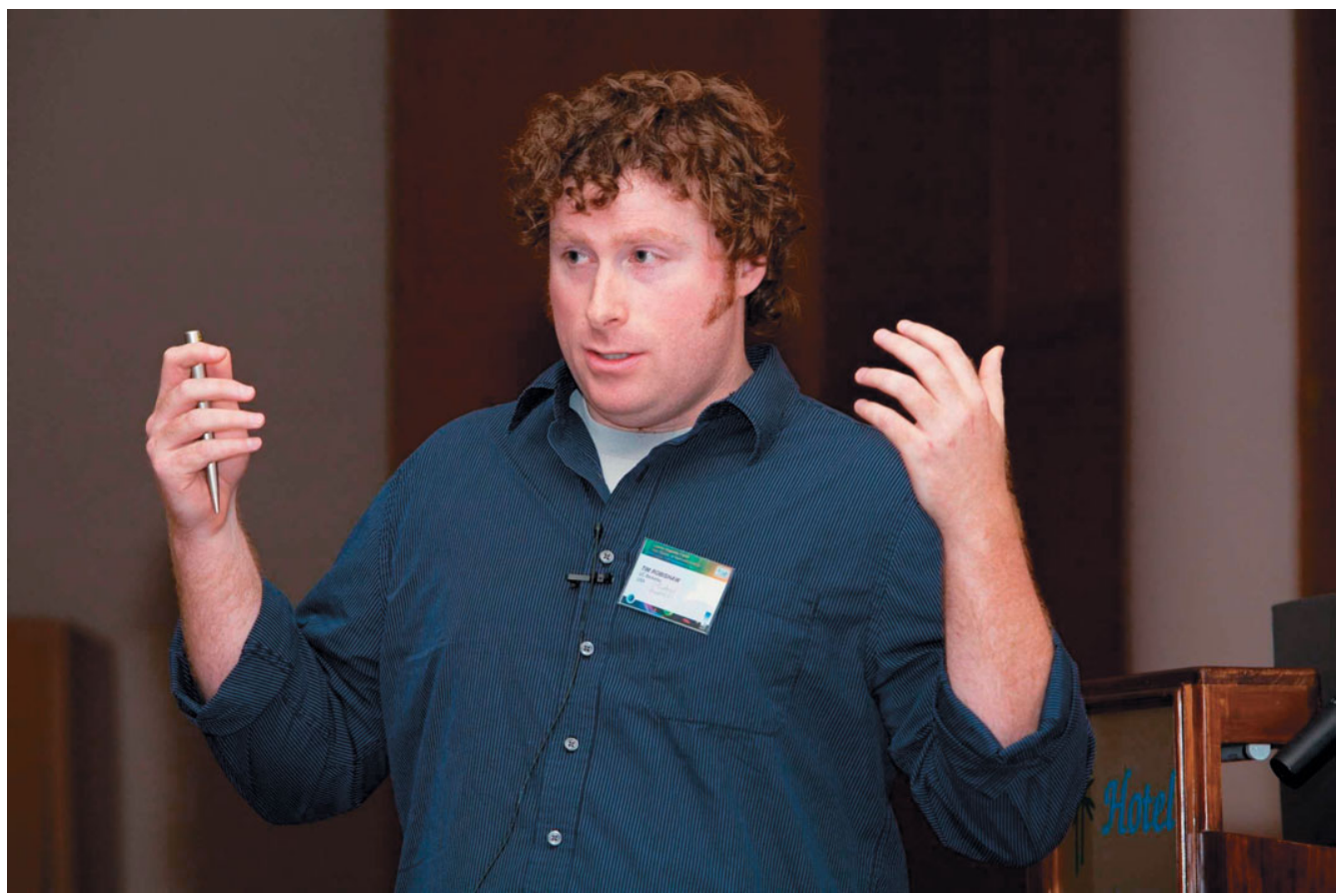

Timothy Robishaw

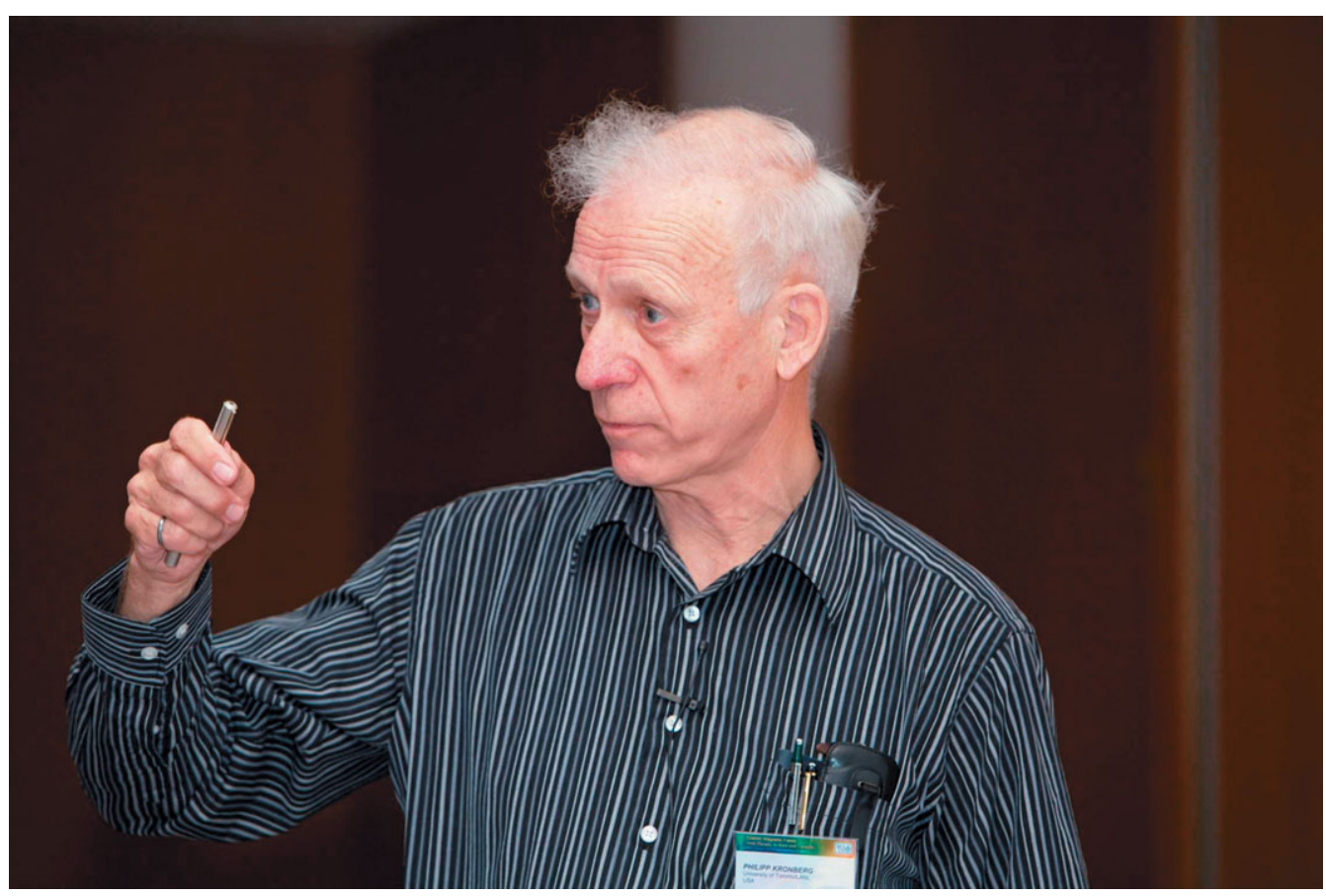

Philipp Kronberg 